\title{
Microstructural white matter alterations in psychotic disorder: A family-based diffusion tensor imaging study
}

Citation for published version (APA):

Domen, P. A. E., Michielse, S., Gronenschild, E., Habets, P., Roebroeck, A., Schruers, K., van Os, J., \& Marcelis, M. (2013). Microstructural white matter alterations in psychotic disorder: A family-based diffusion tensor imaging study. Schizophrenia Research, 146(1-3), 291-300.

https://doi.org/10.1016/j.schres.2013.03.002

Document status and date:

Published: 01/05/2013

DOI:

10.1016/j.schres.2013.03.002

Document Version:

Publisher's PDF, also known as Version of record

Document license:

Taverne

Please check the document version of this publication:

- A submitted manuscript is the version of the article upon submission and before peer-review. There can be important differences between the submitted version and the official published version of record.

People interested in the research are advised to contact the author for the final version of the publication, or visit the DOI to the publisher's website.

- The final author version and the galley proof are versions of the publication after peer review.

- The final published version features the final layout of the paper including the volume, issue and page numbers.

Link to publication

\footnotetext{
General rights rights.

- You may freely distribute the URL identifying the publication in the public portal. please follow below link for the End User Agreement:

www.umlib.nl/taverne-license

Take down policy

If you believe that this document breaches copyright please contact us at:

repository@maastrichtuniversity.nl

providing details and we will investigate your claim.
}

Copyright and moral rights for the publications made accessible in the public portal are retained by the authors and/or other copyright owners and it is a condition of accessing publications that users recognise and abide by the legal requirements associated with these

- Users may download and print one copy of any publication from the public portal for the purpose of private study or research.

- You may not further distribute the material or use it for any profit-making activity or commercial gain

If the publication is distributed under the terms of Article $25 \mathrm{fa}$ of the Dutch Copyright Act, indicated by the "Taverne" license above, 


\title{
Microstructural white matter alterations in psychotic disorder: A family-based diffusion tensor imaging study
}

\author{
Patrick A.E. Domen ${ }^{a, *}$, Stijn Michielse ${ }^{\mathrm{a}, 1}$, Ed Gronenschild ${ }^{\mathrm{a}, 1}$, Petra Habets ${ }^{\text {a }}$, Alard Roebroeck ${ }^{\mathrm{c}}$, \\ Koen Schruers ${ }^{a}$, Jim van Os ${ }^{\text {a,b }}$, Machteld Marcelis ${ }^{a}$ for G.R.O.U.P. \\ a Department of Psychiatry and Psychology, South Limburg Mental Health Research and Teaching Network, EURON, Maastricht University Medical Centre, The Netherlands \\ b King's College London, King's Health Partners, Department of Psychosis Studies, Institute of Psychiatry, United Kingdom \\ ' Department of Cognitive Neuroscience, Maastricht University, The Netherlands
}

\section{A R T I C L E I N F O}

\section{Article history:}

Received 15 June 2012

Received in revised form 26 February 2013

Accepted 1 March 2013

Available online 22 March 2013

\section{Keywords:}

Schizophrenia

Diffusion tensor imaging

White matter

Fractional anisotropy

Healthy siblings

Intermediate phenotype

\begin{abstract}
A B S T R A C T
Background: There is evidence for microstructural white matter alterations in patients with psychotic disorder, suggesting altered interregional connectivity. Less is known about the presence and role of white matter alterations in well individuals at higher than average genetic risk for psychotic disorder.

Methods: 85 patients with psychotic disorder, 93 non-psychotic siblings of patients with psychotic disorder and 80 healthy controls underwent a diffusion tensor imaging (DTI) scanning protocol. In a whole brain voxel-based analysis using Tract Based Spatial Statistics (TBSS), fractional anisotropy (FA) values were compared between the three groups. Effects of antipsychotic medication and drug use were examined.

Results: The patients displayed significantly lower mean FA than the controls in the following regions: corpus callosum (genu, body, splenium), forceps major and minor, external capsule bilaterally, corona radiata (anterior, posterior) bilaterally, left superior corona radiata and posterior thalamic radiation bilaterally. Similar FA differences existed between the patients and siblings; the siblings did not differ from the controls.

Conclusion: Profound microstructural white matter alterations were found in the corpus callosum and other tracti and fasciculi in the patients with psychotic disorder, but not in siblings and the controls. These alterations may reflect brain pathology associated with the illness, illness-related environmental risk factors, or its treatment, rather than genetic risk.
\end{abstract}

(C) 2013 Elsevier B.V. All rights reserved.

\section{Introduction}

There is growing evidence that cerebral vulnerability in schizophrenia may be mediated by altered connectivity between brain regions, rather than focal brain alterations. Indeed, neurophysiological and functional neuroimaging studies have demonstrated pathological functional connectivity (Friston and Frith, 1995; Andreasen et al., 1998; Konrad and Winterer, 2008). Dysconnectivity, in terms of impaired axonal mechanisms and/or abnormal control of synaptic plasticity, may form the core pathology of schizophrenia (Friston, 1998), and may be based in structural alterations. In support of this, volumetric MRI studies have shown decreased white matter volumes of the frontal lobes and temporo-parietal regions and a decreased corpus callosum volume (genu and/or truncus) in schizophrenia patients (Walterfang et al., 2006; Makris et al., 2010; Olabi et al., 2011). Since the late nineties, numerous DTI-studies in patients with a diagnosis of

\footnotetext{
* Corresponding author at: Department of Psychiatry and Psychology, Maastricht University Medical Centre, P.O. Box 616, location Vijverdal, 6200 MD Maastricht, The Netherlands. Tel.: + 3143388 3968; fax: + 31433884122

E-mail address: p.domen@maastrichtuniversity.nl (P.A.E. Domen).

1 Contributed equally.
}

schizophrenia have been published, showing a decrease in FA, indicative of white matter integrity loss, in several brain tracts, including the fronto-temporal connections, such as the arcuate fasciculus, anterior cingulum bundle, uncinate fasciculus (Burns et al., 2003; Kanaan et al., 2005; Kubicki et al., 2007) and fronto-occipital tracts (Ardekani et al., 2003; Mitelman et al., 2007). A meta-analysis in 2009 concluded that significant reductions were present in frontal deep white matter (genu corpus callosum, cingulum bundle, left anterior thalamic radiation, left corticobulbar tract and left inferior fronto-occipital fasciculus) and temporal deep white matter (splenium corpus callosum, fornix/stria terminalis, left inferior longitudinal fasciculus and left inferior fronto-occipital fasciculus) (Ellison-Wright and Bullmore, 2009). Although most, but not all studies (Steel et al., 2001; Hubl et al., 2004; Price et al., 2005) report FA decreases associated with schizophrenia, there is inconsistency regarding the location of the affected brain regions, which, in part, may be related to differences in scanning protocol, study design (e.g. characteristics of the participants) and analytical techniques.

Structural dysconnectivity in patients with schizophrenia may reflect disease-related pathology, but may also represent expression of genetic risk for the disorder (Marenco and Radulescu, 2010). Thus, volumetric MRI studies in first-degree relatives (McDonald 
et al., 2004; Goghari et al., 2007) and twins (Hulshoff Pol et al., 2006) have shown indirect genetic effects on global gray and white matter volume reduction in schizophrenia. The number of DTI studies examining individuals at higher than average genetic risk for psychotic disorder is scant and sample sizes are, in general, small (number of high-risk individuals ranging from $n=16$ to $n=34$ ), except for one study (Boos et al., 2012). The available evidence suggests that white matter alterations may be present in first-degree relatives without symptoms (Munoz Maniega et al., 2008; Camchong et al., 2009; Hao et al., 2009; Narr et al., 2009; Clark et al., 2011; Boos et al., 2012; Knochel et al., 2012). The results for so-called "ultra-high risk" samples with (pre)clinical symptoms are conflicting (Peters et al., 2010), which likely is related to lack of consistency in ultra-high risk sample enrichment procedures across studies (van Os and Linscott, 2012) as well as to differences in brain regions studied and methodological approaches, precluding definite conclusions.

In the present large DTI study ( $\mathrm{n}=258$ ), whole-brain, voxel-based analytic techniques were used to examine patients with a psychotic disorder (highest genetic risk group), non-psychotic siblings (higher than average genetic risk group) of patients with a psychotic disorder, and healthy controls (average genetic risk group). We hypothesized that the patients with psychotic disorder would show reduced white matter integrity compared to the healthy controls, particularly in the corpus callosum, fronto-temporal, and fronto-parietal connections, with the siblings showing a pattern of alterations of intermediate severity.

\section{Methods}

\subsection{Participants}

The patients were recruited from an on-going longitudinal MRI study in Maastricht, the Netherlands. In selected representative geographical areas in the Netherlands and Belgium, the patients were identified through representative clinicians providing health care for patients with psychotic disorder. The siblings were contacted through the participating patients. Mailings and advertisements were effectuated in local newspapers of the same geographical area in order to recruit control participants. The total sample consisted of 258 participants: 85 patients with a psychotic disorder, 93 siblings without a psychotic disorder and 80 healthy controls. The sample included 56 families, of which 35 families contributed one patient and one healthy sibling, three families contributed one patient and two healthy siblings, and one family contributed one patient and three healthy siblings. One family contributed two patients, six families contributed two healthy siblings, and one family contributed three healthy siblings. In the control group, 9 families contributed two siblings. In addition, 44 families contributed a single patient, 34 families contributed a single sibling, and 62 families contributed a single control.

Inclusion criteria were: age range $16-50$ years a good command of Dutch language and for patients: a diagnosis of non-affective psychotic disorder with illness duration of $<10$ years. The siblings and the controls did not have a lifetime diagnosis of any non-affective psychotic disorder. In addition, the controls had no first-degree relative with a lifetime diagnosis of any psychotic disorder, assessed using the Family Interview for Genetic Studies (FIGS) (Maxwell, 1992).

Diagnosis was based on the Diagnostic and Statistical Manual of Mental Disorder-IV (DSM-IV) criteria (APA, 2000), measured with the Comprehensive Assessment of Symptoms and History (CASH) interview (Andreasen et al., 1992). The patients were diagnosed as follows: schizophrenia $(n=59)$, schizoaffective disorder $(n=9)$, schizophreniform disorder $(n=4)$, brief psychotic disorder $(n=2)$, and psychotic disorder not otherwise specified $(n=11)$. Psychopathology in the siblings and controls was also assessed and respectively 18 and 12 participants had a history of a major depressive disorder. None of these met the criteria for a current depressive episode.
All the participants were screened before MRI acquisition for the following exclusion criteria: brain injury with unconsciousness of greater than $1 \mathrm{~h}$, meningitis or other neurological diseases with possible impact on brain structure or function, cardiac arrhythmia requiring medical treatment and severe claustrophobia. In addition, subjects with metal corpora aliena were excluded from the study, as were women with intrauterine device status and (suspected) pregnancy.

The standing ethics committee approved the study protocol, and all the participants gave written informed consent in accordance with the committee's guidelines.

\subsection{Measures}

Level of psychotic symptomatology at the time of scanning was assessed with the Positive and Negative Symptom Scale (PANSS) (Kay et al., 1987) in all three groups. The five factor model by van der Gaag et al. (2006), was used dividing the PANSS in positive symptoms, negative symptoms, disorganization symptoms, excitement and emotional distress. The scores of the individual items of the 5 symptom dimensions were summed.

Educational level was defined as highest accomplished level of education. Handedness was assessed using the Annett Handedness Scale (Annett, 1970).

In the patient group, antipsychotic (AP) medication use was determined by the patient's report and verified with the treating consultant psychiatrist. Best estimate lifetime (cumulative) AP use was determined by multiplying the number of days of AP use with the corresponding haloperidol equivalents and summing these scores for all periods of AP use (including the exposure period between baseline assessment for the G.R.O.U.P. study and the moment of baseline MRI scanning), using the recently published converting formulas for AP dose equivalents described by Andreasen et al. (2010).

Substance use was measured with the Composite International Diagnostic Interview (CIDI) sections B-J-L (WHO, 1990). Use of cannabis and other drugs was assessed as reported frequency of use during the last 12 months, as well as lifetime use. CIDI frequency data on lifetime cannabis and other drug use was available for respectively 250 participants (3\% missing data) and 256 participants (1\% missing data).

Alcohol use was defined as the reported number of weekly consumptions during the last 12 months.

\subsection{Image acquisition}

Magnetic resonance imaging scans were obtained at Maastricht University, the Netherlands, using an Allegra syngo MR A30 (Siemens, Erlangen, Germany) operating at 3.0 T. The following anatomical scan parameters were used: Modified Driven Equilibrium Fourier Transform (MDEFT) sequence; 176 slices, $1 \mathrm{~mm}$ isotropic voxel size, echo time $2.4 \mathrm{~ms}$, repetition time $7.92 \mathrm{~ms}$, inversion time $910 \mathrm{~ms}$, flip angle $15^{\circ}$, total acquisition time $12 \mathrm{~min}$ and $51 \mathrm{~s}$; Magnetization Prepared Rapid Acquisition Gradient-Echo (MPRAGE; Alzheimer's Disease Neuroimaging Initiative) sequence 192 slices, $1 \mathrm{~mm}$ isotropic voxel size, echo time $2.6 \mathrm{~ms}$, repetition time $2250 \mathrm{~ms}$, inversion time $900 \mathrm{~ms}$, flip angle $9^{\circ}$, total acquisition time $7 \mathrm{~min}$ and $23 \mathrm{~s}$. The matrix size was $256 \times 256$ and field of view was $256 \times 256 \mathrm{~mm}^{2}$. The number of excitations was one. Two sequences were used because of a scanner update during data collection.

Microstructural anatomy was examined using diffusion tensor imaging with an echo-planar-imaging sequence (field of view $230 \times$ $230 \mathrm{~mm}^{2}$, TR $10,800 \mathrm{~ms}$, TE $84 \mathrm{~ms}$, voxel size $1.8 \times 1.8 \times 1.8 \mathrm{~mm}^{3}$, b-value $1000 \mathrm{~s} / \mathrm{mm}^{2}$, noise level 40, 85 slices, no overlap). As a result of the scanner update, two DTI sequences were used: one with 76 directions ( of which 4 T2-weighted (B0) and 72 diffusion-weighted (B)), and one with 81 directions $(8 \times \mathrm{B} 0$ and $73 \times \mathrm{B})$. The proportion of scans with 76 directions was balanced between the groups (78\% in the controls, 
$75 \%$ in the siblings and $69 \%$ in the patients $\left(\chi^{2}=1.52, \mathrm{P}=0.468\right)$, preventing any systematic bias. Total acquisition time of the DTI sequence was $15 \mathrm{~min}$.

\subsection{Diffusion tensor imaging analysis}

Processing of DTI data was effectuated using tract-based spatial statistics (TBSS) v1.2 in FSL 4.1.6 (FMRIB Analysis Group, Oxford, UK, http://www.fmrib.ox.ac.uk/analysis/research/tbss). First, standard Siemens DICOM files were transformed into compressed NIFTI format using a custom built in-house software named GIANT (General Image ANalysis Tools developed by EHBMG). Raw data were corrected for head movement and eddy currents invoked during scanning. The B0 volume was skull-stripped using FSL's Brain Extraction Tool (Smith, 2002) and this served as a brain mask for all B volumes.

The next step was fitting a diffusion tensor model at each voxel using data output from the brain extraction, diffusion weighted data and gradient directions following a general linear model (FreeSurfer v4.5.0, http://surfer.nmr.mgh.harvard.edu). After tensor fitting the process continued working on FA volumes, eroding them slightly.

Nonlinear registration aligned each FA volume to $1 \times 1 \times 1 \mathrm{~mm}$ standard FMRIB58_FA space. The standard FMRIB58_FA contains a template derived from high-resolution images of 58 participants in a well-aligned population (both males and females ranging between 20 and 50 years of age) (Smith et al., 2006).

After nonlinear transformation of the FA volumes into standard space, a mean FA skeleton from all the participants per group was derived. The mean FA skeleton follows the major white matter tracts in each individual participant (normalized in MNI152 space) and provides a way to compare between (groups of) the participants. The final step of the processing was setting the FA threshold using visual inspection of the FA skeleton, in the present study at a level of 0.25 , to include major white matter tracts while removing small peripheral tracts that would cause excess inter-participant variability. In addition, this threshold setting avoided inclusion of regions that are likely to be composed of multiple tissue types or fiber orientations.

\subsection{Statistical analyses}

Voxel-wise statistical analysis was performed on the mean FA skeleton using a general linear model and applying FSL's randomize (v2.1) permutation-testing script (Smith et al., 2004). The comparisons involved the statistical tests of group differences in FA, yielding a total of six contrasts: i) mean FA is lower (or higher) in the patients compared to the controls, ii) mean FA is lower (or higher) in the siblings compared to the controls, and iii) mean FA is lower (or higher) in the patients compared to the siblings. The a priori hypothesized confounding variables age, sex, handedness, level of education, lifetime cannabis use and lifetime other drug use were used in the statistical model. In addition to tests for regional group differences, stratified gender analyses were carried out.

In addition, associations between AP medication and FA were analyzed in two different ways. First, cumulative AP exposure was added as an additional covariate. Second, in the patients only, cumulative AP exposure was entered as independent (dummy) variable representing the distribution of scores divided by its tertiles (low, medium and high exposure) to examine the effect on FA within the patients.

Lastly, planned sensitivity analyses were performed excluding the siblings and controls with a history of affective disorder.

For the main analysis permutation tests were performed using 50,000 permutations in order to reduce the margin of error to acceptable uncertainty levels ( $P=0.05 \pm 0.00195$, i.e. $3.9 \%$ of the nominal alpha). With regard to the additional analyses (effects of AP medication and gender, exclusion of affective disorder) 10,000-50,000 permutations were performed. The threshold free cluster enhancement
(TFCE) option was enabled to find clusters without setting an initial cluster level (Smith and Nichols, 2009). Statistical maps (thresholded at $\mathrm{P}<0.05$ ) were used for assessing differences between groups (corrected for multiple comparisons).

The Johns Hopkins University International Consortium for Brain Mapping (JHU ICBM)-DTI-81 white-matter atlas labels (Mori et al., 2008) were used to label significant voxels and assign a specific tract name. If the voxels did not match with the JHU ICBM labels, they were identified using the JHU white-matter tractography atlas (Hua et al., 2008). A binary mask was created containing the significant clusters in order to extract FA values of the individual participants.

For visualization purposes (see Fig. 1) and to extend the TBSS analyses, from all $38 \mathrm{JHU}$ labeled white matter tracts, skeleton mean FA values were extracted and exported to Stata version 12 (StataCorp, 2009). Regional group differences were tested with multilevel random regression procedures using the Stata XTREG command with adjustment for the a priori hypothesized confounding variables age, sex, handedness, and level of education.

In addition, the data set was transformed from a wide to a long format, resulting in a hierarchically structured data set, with 38 regional FA measures (Level 1) nested in subjects (Level 2) who were part of the same families (Level 3). Because of the three-level grouping structure of the data, compromising statistical independence of the observations, multilevel random regression models were fitted using the XTMIXED command in Stata with FA measures as the dependent variable and subject number and family number modeled as random effects. Mixed models contain both fixed and random effects, the fixed effects being analogous to standard regression coefficients (B).

Main effects of group and group $\times$ sex interactions in the model of FA were examined, as well as associations between cumulative AP dose and FA in the patient group. The same covariates were used as described above.

\section{Results}

\subsection{Descriptive analyses}

There were more women than men in the control group, whereas the opposite held for the patient group. The study comprised a relatively stable patient group as reflected by the low PANSS scores (Table 1 ). The patients were more frequent cannabis- and other drug users than the siblings and controls (Table 1). At the time of scanning, seventy patients were receiving AP medication (second generation: $n=67$; first generation: $n=3$ ). The mean current dosage of AP medication in terms of standard haloperidol equivalents was 5.5 milligrams $(\mathrm{mg})(\mathrm{SD}=4.6)$. Furthermore, 16 patients used antidepressants, 6 used benzodiazepines, 5 used anticonvulsants, and 2 used lithium. Three siblings and 3 control participants used antidepressants, and one control participant used benzodiazepines.

\subsection{Whole brain group differences in FA}

Voxel-wise analysis in TBSS revealed significant differences in mean FA in two out of six comparisons, indicating that the patients had a lower mean FA than the controls and siblings. The number of voxels for each white matter tract with a significant higher $F A(P<0.05)$ in the controls and siblings compared to the patients, was derived and shown in Table 2.

The patients showed a significantly lower FA compared to the control group in the following regions with $>250$ voxels and thresholded at $\mathrm{P}<0.05$ : corpus callosum (genu, body, splenium), forceps major and minor, external capsule bilaterally, corona radiata (anterior, posterior) bilaterally, left superior corona radiata and posterior thalamic radiation bilaterally. At a more conservative threshold $(\mathrm{P}<0.01)$, the 

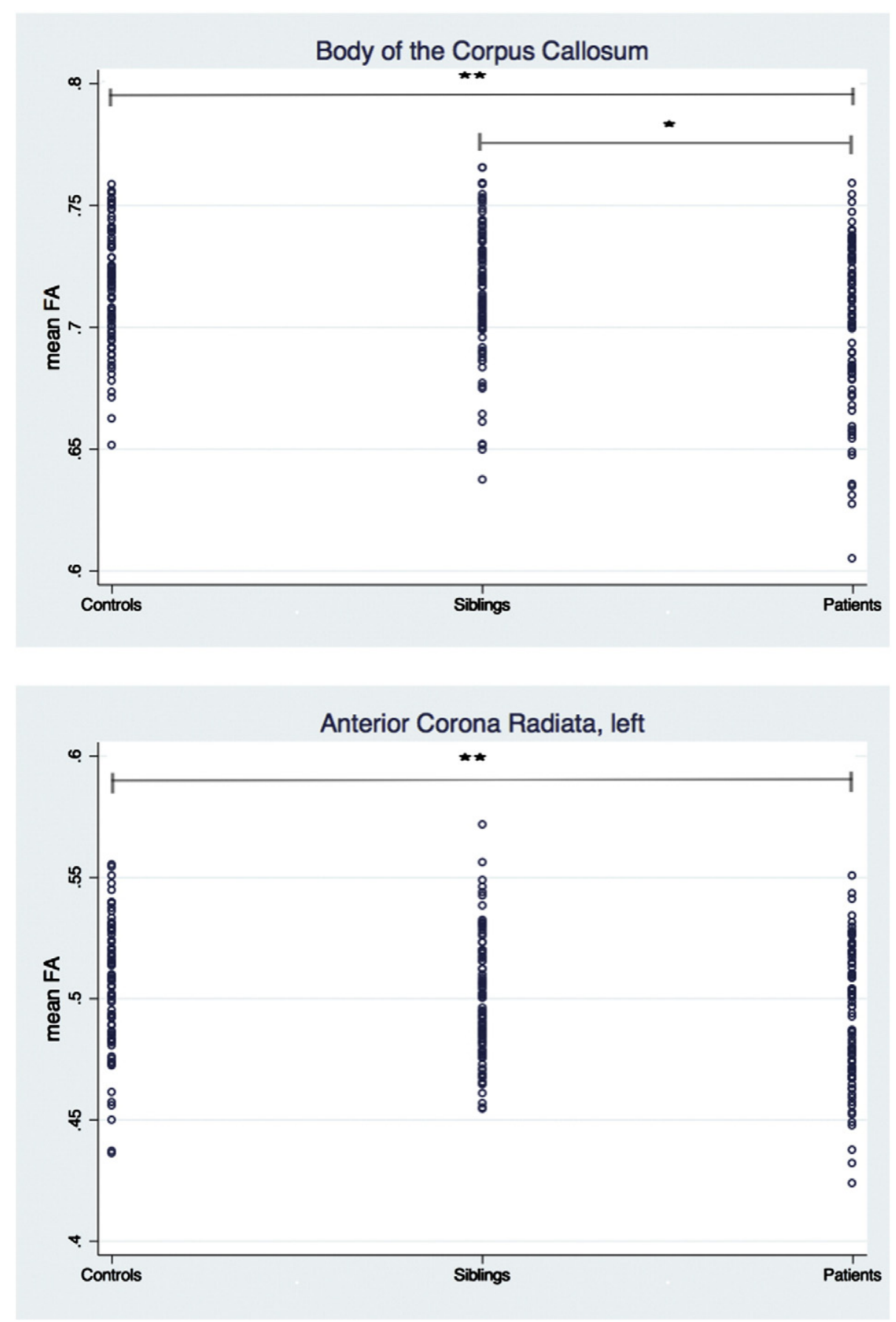

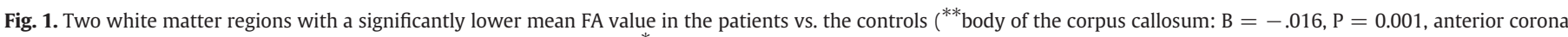

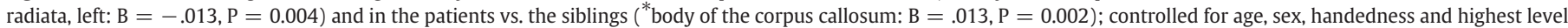
of education.

corpus callosum (genu, body), left external capsule en anterior corona radiata bilaterally remained significant (Fig. 2).

The mean FA values in the brain areas mentioned above showed a gradual decline from the controls to the siblings to the patients (see Fig. 1, results from multilevel random regression procedures in Stata). Although mean FA values in the siblings were generally lower than FA values in the controls, these differences were neither large nor conclusive statistically.

Compared to the siblings, the patients showed a significantly lower FA in the following regions with $>250$ voxels and thresholded at $\mathrm{P}<0.05$ : corpus callosum (genu, body), forceps minor, left anterior corona radiata, right superior and posterior corona radiata and the right posterior thalamic radiation. Only a few regions remained significant using a more conservative threshold, i.e. $\mathrm{P}<0.01$ : the right body of the corpus callosum and the right superior corona radiata (Fig. 2). All these regions overlapped with those standing out in the comparison between patients and healthy controls.

There were no regions with a higher mean FA in the patients compared to the controls or with a higher mean FA in the siblings compared to the controls.

\subsection{Additional covariates: cannabis and other drug use}

The results described above were controlled for age, sex, handedness, and highest level of education. The analyses were repeated using the additional covariates of cannabis and other drug use. When only 
Table 1

Demographic characteristics of the participants.

\begin{tabular}{|c|c|c|c|}
\hline & Controls $(\mathrm{n}=80)$ & Siblings $(\mathrm{n}=93)$ & Patients $(\mathrm{n}=85)$ \\
\hline Sex, male (\%) & $29(36 \%)$ & $49(53 \%)$ & $58(68 \%)$ \\
\hline Handedness & 76.3 & 73.9 & 72.8 \\
\hline Age at scan (years) & $30.8 \pm 10.8$ & $29.4 \pm 8.8$ & $28.3 \pm 7.0$ \\
\hline $\begin{array}{l}\text { Level of education } \\
\text { Age of onset (years) }\end{array}$ & $5.4 \pm 1.8$ & $5.1 \pm 2.1$ & $\begin{array}{l}4.1 \pm 2.0 \\
22.8 \pm 6.4\end{array}$ \\
\hline $\begin{array}{l}\text { Illness duration } \\
\text { (years) }\end{array}$ & - & - & $5.4 \pm 3.6$ \\
\hline Antipsychotics ${ }^{\mathrm{a}}$ & - & - & $6692.71 \pm 6254.18$ \\
\hline Cannabis $^{\mathrm{b}}$ & $7.8 \pm 21.9$ & $19.3 \pm 37.2$ & $44.0 \pm 47.0$ \\
\hline Other drugs ${ }^{\mathrm{b}}$ & $0.90 \pm 4.7$ & $6.2 \pm 31.4$ & $42.4 \pm 90.8$ \\
\hline Alcohol $^{\mathrm{C}}$ & $5.0 \pm 7.0$ & $9.8 \pm 17.3$ & $5.0 \pm 9.1$ \\
\hline \multicolumn{4}{|l|}{ PANSS } \\
\hline Positive symptoms & $7.3 \pm 1.1$ & $7.3 \pm 0.9$ & $10.4 \pm 5.0$ \\
\hline Negative symptoms & $8.2 \pm 1.0$ & $8.4 \pm 2.0$ & $12.0 \pm 5.9$ \\
\hline Disorganization & $10.2 \pm 1.2$ & $10.3 \pm 0.7$ & $12.5 \pm 4.1$ \\
\hline Excitement & $8.3 \pm 1.1$ & $8.6 \pm 1.4$ & $9.7 \pm 2.7$ \\
\hline Emotional distress & $9.2 \pm 2.1$ & $9.9 \pm 2.6$ & $13.2 \pm 5.2$ \\
\hline
\end{tabular}

Means \pm SDs are reported.

Abbreviations: PANSS, Positive and Negative Syndrome Scale.

a Lifetime exposure in haloperidol equivalents.

b Mean number of times; life time.

c Weekly consumptions on the last 12 months.

cannabis use was added as an additional covariate, the number of significant brain regions showing FA difference between the patients and the controls, as well as the number of voxels per region was comparable to the analysis without cannabis use as a covariate. The right superior longitudinal fasciculus became apparent as an additional area with a significant higher FA in the controls than in the patients (see Table 2).

When both cannabis and other drugs were added as covariates, the number of white matter areas with significant FA differences between the patients and the controls slightly decreased, particularly on the right side of the brain. Effect sizes of the genu and body of the corpus callosum held, whereas the effect size of the splenium was decreased (Table 2). Overall, the effect of drug use on white matter tissue appeared to be minor in this sample.

\subsection{AP medication}

When cumulative AP exposure was added in TBSS as an additional covariate, the number of significant voxels decreased markedly, but significantly lower FA in the patients compared to the controls remained present in the body of the corpus callosum and the right posterior corona radiata ( $>250$ voxels). The patient-sibling comparison did not show significant regional differences.

Cumulative AP exposure was also entered as an independent (dummy) variable (low exposure: $n=22$, medium exposure: $n=22$ and high exposure: $\mathrm{n}=21$ ) to examine the effect on FA within the patients. No significant differences in mean FA values were found when high or medium AP exposure levels were compared to low AP exposure levels.

In addition, analysis in Stata showed that there was no significant association between lifetime AP use and FA ( $\left.\mathrm{B}=1.4 \times 10^{-7}, \mathrm{P}=0.68\right)$.

\subsection{Sex differences}

Stratified TBSS analyses were done separating the male $(n=29$ controls, 49 siblings, 58 patients) and the female subjects ( $\mathrm{n}=51$ controls, 44 siblings, 27 patients). This led to $50 \%$ reduction in sample size and to an absence of significant regional differences in mean FA in the patient-control, patient-sibling and control-sibling comparisons.

Since the number of the participants varied considerably between the groups after separating them by sex (e.g. far more male patients than controls, and vice versa for females), the analyses were repeated in equally sized groups, i.e. three groups of 29 males and three groups
Table 2

Group differences in FA: results from TBSS.

\begin{tabular}{|c|c|c|c|c|}
\hline \multirow[t]{2}{*}{ Brain Area } & \multirow{2}{*}{$\begin{array}{l}\text { Siblings vs. } \\
\text { patients } \\
\text { A }\end{array}$} & \multicolumn{3}{|c|}{ Controls vs. patients } \\
\hline & & A & $\mathrm{B}$ & $\mathrm{C}$ \\
\hline Genu of corpus callosum & 826 & 841 & 928 & 800 \\
\hline Body of corpus callosum & 1983 & 2421 & 2581 & 2380 \\
\hline Splenium of corpus callosum & 92 & 346 & 412 & 165 \\
\hline Forceps major & 94 & 276 & 326 & 122 \\
\hline Forceps minor & 1068 & 1038 & 1149 & 984 \\
\hline Fornix (column and body of fornix) & 0 & 0 & 0 & 0 \\
\hline Anterior limb of internal capsule, right & 0 & 49 & 398 & 0 \\
\hline Anterior limb of internal capsule, left & 0 & 213 & 48 & 0 \\
\hline Posterior limb of internal capsule, right & 0 & 0 & 22 & 0 \\
\hline Posterior limb of internal capsule, left & 0 & 1 & 0 & 0 \\
\hline Retrolenticular part of internal capsule, right & 0 & 4 & 2 & 0 \\
\hline Retrolenticular part of internal capsule, left & 0 & 0 & 1 & 1 \\
\hline Anterior corona radiata, right & 0 & 1037 & 1049 & 750 \\
\hline Anterior corona radiata, left & 654 & 1157 & 1233 & 1120 \\
\hline Superior corona radiata, right & 395 & 241 & 394 & 175 \\
\hline Superior corona radiata, left & 221 & 468 & 470 & 382 \\
\hline Posterior corona radiata, right & 339 & 373 & 406 & 0 \\
\hline Posterior corona radiata, left & 13 & 442 & 443 & 416 \\
\hline $\begin{array}{l}\text { Posterior thalamic radiation } \\
\text { (incl. optic radiation), right }\end{array}$ & 253 & 244 & 255 & 0 \\
\hline $\begin{array}{l}\text { Posterior thalamic radiation } \\
\text { (incl. optic radiation), left }\end{array}$ & 0 & 313 & 352 & 260 \\
\hline $\begin{array}{l}\text { Sagittal stratum (incl. inf. longitudinal fasc. } \\
\text { and inferior fronto-occ. fasc), right }\end{array}$ & 149 & 3 & 8 & 0 \\
\hline $\begin{array}{l}\text { Sagittal stratum (incl. inf. longitudinal fasc. } \\
\text { and inferior fronto-occ. fasc), left }\end{array}$ & 0 & 5 & 46 & 35 \\
\hline External capsule, right & 0 & 255 & 394 & 0 \\
\hline External capsule, left & 0 & 371 & 425 & 400 \\
\hline Cingulum (cingulate gyrus), right & 0 & 57 & 140 & 127 \\
\hline Cingulum (cingulate gyrus), left & 23 & 0 & 0 & 0 \\
\hline Cingulum (hippocampus), right & 0 & 0 & 0 & 0 \\
\hline Cingulum (hippocampus), left & 0 & 0 & 0 & 0 \\
\hline Fornix, stria terminalis, right & 0 & 19 & 27 & 0 \\
\hline Fornix, stria terminalis, left & 0 & 4 & 18 & 16 \\
\hline Superior longitudinal fasciculus, right & 0 & 0 & 269 & 0 \\
\hline Superior longitudinal fasciculus, left & 0 & 184 & 198 & 173 \\
\hline Superior fronto-occipital fasciculus, right & 103 & 65 & 69 & 0 \\
\hline Superior fronto-occipital fasciculus, left & 0 & 64 & 62 & 0 \\
\hline Uncinate fasciculus, right & 0 & 50 & 57 & 0 \\
\hline Uncinate fasciculus, left & 0 & 53 & 58 & 56 \\
\hline Tapetum, right & 0 & 35 & 35 & 0 \\
\hline Tapetum, left & 0 & 5 & 5 & 5 \\
\hline
\end{tabular}

The number of voxels with a significant higher FA $(P<0.05)$ in the controls vs. the patients and the siblings vs. the patients. There were no regions with a significant higher FA in the siblings compared to the controls. Results were controlled (A) for age, sex, handedness, highest level of education, (B) age, sex, handedness, highest level of education and cannabis use, (C) age, sex, handedness, highest level of education, cannabis and other drug use.

of 27 females. The total number of significant voxels markedly decreased: $8 \%$ (=1291 voxels) left in the female patient-control comparison, and $28 \%$ (=1845 voxels) left in the male patient-sibling comparison. In females, the patient group showed significantly lower FA values compared to the controls, but not compared to the siblings, in the genu and body of the corpus callosum and the forceps minor (>250 voxels). In males, the patient group showed significantly lower mean FA values compared to the siblings, but not compared to the controls, following a similar regional pattern as in the whole group analysis, with only the body of the corpus callosum exceeding 250 voxels. There were no significant mean FA differences in the sibling-control comparisons.

In addition, although there was a significant main effect of group in the model of FA (group, linear trend: $\mathrm{B}=-0.004, \mathrm{P}=0.02$; patients compared to controls: $\mathrm{B}=-0.007, \mathrm{P}=0.02$; siblings compared to controls: $\mathrm{B}=-0.002, \mathrm{P}=0.45)$, the group $\times$ sex interaction was not significant (chi ${ }^{2}: 1.04, P=0.60$ ), suggesting that the effect of group is not conditional on sex. 


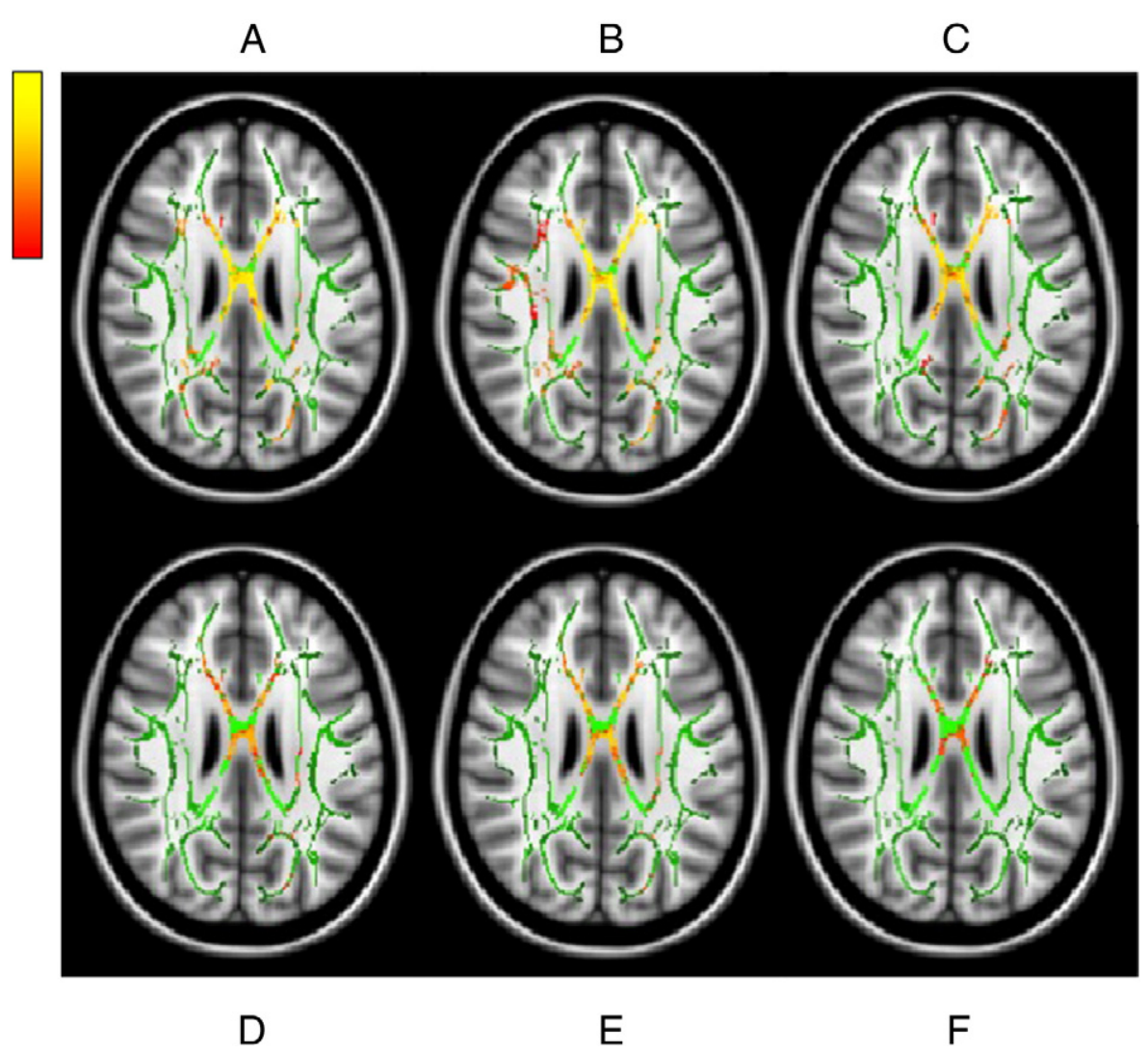

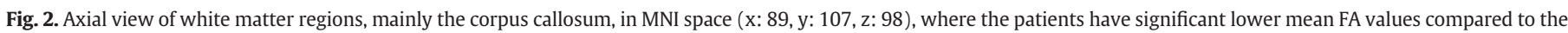

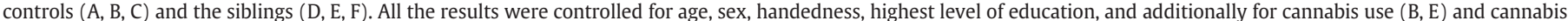
and other drug use $(C, F)$. Statistical maps were thresholded at $\mathrm{P}<0.05$, with the colormap reflecting the P-values, ranging from 0.05 (red) to 0.00 (yellow).

\subsection{Exclusion of individuals with a history of affective disorder}

Repeating the original analyses excluding siblings ( 8 males and 10 females) and controls ( 1 male and 11 females) with a history of affective disorder resulted in an overall increase $(+15 \%)$ of significant voxels in the patient-control comparison, and an overall decrease $(-76 \%)$ of significant voxels in the patient-sibling comparison. The regional pattern of patient-related decreased FA was not changed, except for the absence of the left anterior, superior and posterior corona radiata in the patient-sibling comparison. No significant differences in mean FA values were found between the controls and the siblings.

\section{Discussion}

In this whole-brain DTI study, patients with a psychotic disorder, non-psychotic siblings and healthy controls were examined in order to identify microstructural white matter alterations that may be associated with the familial risk for schizophrenia. The results showed a gradual decline of mean FA from the controls to the siblings to the patients. Mean FA values were significantly lower in the patients compared to the controls in several major white matter tracts, while differences between the siblings and the controls did not reach statistical significance. Thus, microstructural white matter alterations may reflect brain pathology associated with the illness, illness related environmental factors, or its treatment, rather than genetic risk.

\subsection{Findings in the patients}

The results support the hypothesis that the patients with psychotic disorder compared to the healthy controls have reduced connectivity in several brain regions, while no regions with increased FA were identified. This is in line with the majority of the previous evidence suggesting widespread FA decreases in schizophrenia (Kubicki et al., 2007; Ellison-Wright and Bullmore, 2009; Peters et al., 2010). A significantly lower mean FA in the patients in certain brain fasciculi and tracts may indicate dysfunctional axonal connectivity, which has been related to psychotic symptomatology. For example, smaller FA in the left superior longitudinal fasciculus has been associated with positive symptoms (Skelly et al., 2008), in particular hallucinations (Seok et al., 2007) and smaller FA in posterior callosal fibers has been associated with negative symptoms (Seok et al., 2007).

In the present study, the most prominent FA decreases were found in the corpus callosum, in particular the anterior part (genu) of the corpus callosum. The corpus callosum and its postulated role in the dysconnectivity hypothesis in schizophrenia are highlighted in several studies, but there is no consensus about which part of the corpus callosum is most affected; the anterior part (Kubicki et al., 2008) or the more posterior interhemispheric connections (Patel et al., 2011). The results from the present study showed that the anterior-middle part was most affected, but also suggest that the entire corpus callosum may in fact be involved. These FA decreases in the corpus callosum provide additional evidence for aberrant cerebral interhemispheric activity in callosal fibers, further strengthening the theory that dysconnection in white matter connectional architecture in this part of the brain plays an important role in the core pathology of schizophrenia (Woodruff et al., 1997; Zalesky et al., 2011). Indeed, aberrant connectivity in the corpus callosum has been associated with social cognition and interhemispheric information transfer deficits (Chaim et al., 2010; Miyata et al., 2010), as well as positive and negative symptoms (Hubl et al., 2004; Rotarska-Jagiela et al., 2008). 


\subsection{Findings in the siblings}

Only a few studies with relatively small samples sizes have examined FA in first-degree relatives of patients with psychotic disorder. Clark et al. (2011) found decreased FA in both patients and nonpsychotic first-degree relatives in the left and right inferior longitudinal fasciculus, the left inferior fronto-occipital fasciculus and the temporal component of the superior longitudinal fasciculus, using a ROI approach (Clark et al., 2011). The effect sizes for the relatives were intermediate to those for the patients and controls. Other studies have reported significant white matter abnormalities in first-degree relatives compared to controls in the prefrontal cortex and hippocampus (Hao et al., 2009), medial frontal regions (Camchong et al., 2009), the anterior limb of the internal capsule (Munoz Maniega et al., 2008) and the corpus callosum (Knochel et al., 2012). One recent family study, comprising larger samples than hitherto, reported absence of FA alterations in most white matter tracts in siblings as compared to patients with schizophrenia and controls, except for an increased FA in the right arcuate fasciculus (Boos et al., 2012).

Although in the present study, the mean FA in the significant areas between the controls and the patients reflected a dose response effect of familial risk in that the values were highest in the controls and lowest in the patients, with intermediate values for the siblings, there were no significant differences between the sibling and the control group. In addition, there were significant changes in the patient versus sibling comparison, suggesting that the siblings are more similar to the controls than the patients. In other words, the present study does not provide strong evidence for a microstructural white matter intermediate phenotype, but rather suggests that the microstructural white matter alterations are contingent on the expression of psychotic illness. As cross-sectional studies do not allow conclusions on direction of effect, fiber dysconnectivity could in fact arise from the disease process or disease-related differential exposure to environmental risk factors or medication use.

\subsection{Antipsychotic medication}

Qualitative reviews have suggested that AP use may affect brain structure (Navari and Dazzan, 2009; Smieskova et al., 2009). A recent, fairly large longitudinal study found that white matter volume loss was associated with more extensive use of antipsychotics even after adjustment for illness duration, illness severity, and substance abuse (Ho et al., 2011). However, cross-sectional DTI studies with patients who were either medication-naïve or briefly medicated did not find evidence for a significant association between FA and antipsychotic exposure (Peters et al., 2008; Szeszko et al., 2008). In the present study, extensive TBSS and Stata analyses both supported absence of significant effects of cumulative AP exposure on FA, except for one specific analysis in TBSS, in which AP exposure was added as a covariate and, consequently, the number of significant voxels between the patients and the controls markedly decreased (by 79\%). This, however, likely is an effect of the randomize procedure in FSL, where all controls and siblings receive a zero value for AP exposure leading to skewed distributions in the equation and distortion of the results (i.e. controlling for AP is like controlling for patient-status itself). Moreover, despite the voxel reduction in this analysis, significantly lower FA in the patients compared to the controls remained present in the body of the corpus callosum and the right posterior corona radiate ( $>250$ voxels), showing the robustness of FA differences in these areas. Lastly, altered FA values have been detected in AP-naïve patients (Cheung et al., 2008; Zou et al., 2008; Gasparotti et al., 2009), suggesting that AP exposure may at best contribute to, but not fully explain, the white matter alterations.

\subsection{Environmental factors}

Specific (epi)genetic or environmental effects and/or geneenvironment interplay may have contributed to the emergence of structural dysconnectivity in patients. Evidence for gene effects has come from studies with healthy subjects and studies with patients showing associations between polymorphisms of myelin-associated genes, such as NRG1, ErbB4 and PIK4CA on the one hand, and white matter integrity in the anterior cingulum, the anterior limb of the internal capsule and frontotemporal fibers on the other (McIntosh et al., 2008; Konrad et al., 2009; Wang et al., 2009; Marenco and Radulescu, 2010; Zuliani et al., 2011).

Certain environmental factors, such as childhood trauma (Andersen et al., 2008) and urban upbringing (Lederbogen et al., 2011) have been associated with cerebral alterations in respectively traumatized and non-psychiatric populations. Although these environments are risk factors for schizophrenia (van Os et al., 2010) they have never been examined in relation to cerebral white matter in this patient population. The influence of cannabis on white matter volume in schizophrenia is weak (Cahn et al., 2004; Solowij et al., 2011) but DTI studies have shown both FA increases and decreases in cannabis-using patients with schizophrenia compared to non-using patients (DeLisi, 2008; Peters et al., 2010). Previous analyses on the present study sample have demonstrated that familial predisposition for psychotic disorder is associated with greater cannabis-induced reduction in cortical thickness (Habets et al., 2010). The current DTI results suggest that there is no marked effect of cannabis on microstructural white matter, as the controlling TBSS analyses for cannabis use did not change the results. However, the number of white matter areas that were significantly different between the patients and the controls slightly decreased in the model that included other drug use as a covariate. This may indicate that non-cannabis drug use may have a subtle, though measurable, effect on microstructural white matter in patients with psychotic disorder. To date, there are no other DTI studies reporting on an association between FA and non-cannabis drug use in individuals with psychotic disorder. In healthy (non-psychotic) individuals, other drug use (cocaine, heroin) has been negatively associated with FA in several brain areas, including the corpus callosum (Lane et al., 2010; Bell et al., 2011; Bora et al., 2012). Thus, other drugs may affect the integrity of white matter brain tracts in individuals with psychotic disorder as well as in healthy (non-psychotic) individuals.

The possible influence of environmental risk factors on white matter tissue warrants further investigation.

\subsection{Sex differences}

Several studies have addressed sex differences in FA in healthy individuals. For the corpus callosum, both higher FA values in women compared to men (Kanaan et al., 2012), higher FA values in men compared to women (Menzler et al., 2011), as well as no sex differences (Sullivan et al., 2001) have been reported. In patients with schizophrenia, the literature on sex differences in FA is limited, but suggests lower values in women compared to men in the genu (Price et al., 2007) and the whole corpus callosum (Rametti et al., 2009). In the present study, the absence of significant group differences in regional FA in males and females separately could be the result of lack of power and markedly unequal group sizes after stratification by sex. Indeed, when stratified analyses were done in equally sized groups, there were patient-control differences in females, but not in males, and sibling-patient differences in males, but not in females. Multilevel analyses in Stata, however, did not yield support for a group $\times$ sex interaction. The contrasting results between TBSS and Stata analyses could be the result of methodological differences (voxel-wise analyses of aligned FA data projected on the mean FA skeleton, versus multilevel regression analyses on the skeleton mean FAs of 38 regions).

In conclusion, the significant FA reductions in patients may be more female than male driven, which needs replication in larger samples. The absence of reduced FA in the siblings compared to the controls, however, was consistent in all analyses, thus consistently providing evidence for the absence of an intermediated phenotype. 


\subsection{Methodological considerations}

Since FA decreases in cortical and subcortical areas have been found in patients with major depression (Liao et al., 2012), a priori planned sensitivity analyses were done excluding all individuals with a history of affective disorder. Although the number of significant voxels varied, which may be due to a different sex distribution between the groups as the majority of the excluded participants were females, it did not alter the regional pattern of significant white matter tracts.

Structural neuroimaging studies often differ in scanning acquisition and imaging processing. These differences could have contributed to discrepancies in outcome. The present study was the second family study using a 3-T scanner. The signal-to-noise ratio using this field strength is better, as is the visibility of certain details compared to a 1.5-T scanner. Also, the sequence alteration due to a scanner update could in theory have influenced our results. However, we repeated the TBSS analyses, with the number of scan directions as additional covariate. The number and pattern of significant findings was not affected (results available on request).

Other methodological differences are reflected in the type of analyses: hypothesis-generating whole-brain voxel-wise analysis versus the hypothesis-testing ROI approach. It is known that due to image misregistration, smoothing and partial volume effects, whole brain voxel-wise analysis often yields different results compared to the region of interest (ROI) studies (Kyriakopoulos et al., 2008; Jones and Cercignani, 2010).

In DTI studies, different atlases are used in establishing the exact regions of white matter alterations. These atlases differ considerably in the definitions of the boundaries of particular white matter tracts and of the labeling of certain brain regions, in an attempt to account for overlap and crossing fibers. We used two versions of the white matter JHU atlas (Hua et al., 2008; Mori et al., 2008) to include most major white matter tracts. The differences in atlases among the DTI studies hamper between-study comparisons.

\section{Conclusion}

This large cross-sectional DTI study examined white matter integrity in relation to different levels of familial risk for psychotic disorder. There was evidence for patient-specific alterations in microstructural white matter, most profoundly present in the corpus callosum, with no evidence for an intermediate phenotype. The widespread disruptions in white matter in patients with schizophrenia may reflect disease-related dysconnectivity, or disease-related differential exposure to environmental risk factors contributing to the symptoms of schizophrenia.

\section{Role of funding source}

This work was sponsored by the Dutch organization for scientific research NWO (Genetic Risk and Outcome of Psychosis [G.R.O.U.P]) and the European Community's Seventh Framework Programme under Grant Agreement No. HEALTH-F2-2009-241909 (European Network of National Schizophrenia Networks Studying Gene-Environment Interactions Consortium).

Both funding sources had no further role in the study design; in the collection, analysis and interpretation of data; in the writing of the report; and in the decision to submit the paper for publication.

\section{Contributors}

M. Marcelis and Jim van Os designed the study and wrote the protocol.

A. Roebroeck contributed to the scanning protocol and preprocessing of the data.

P. Habets and P. Domen collected the data.

E. Gronenschild and P. Habets performed the preprocessing steps.

M. Marcelis, Jim van Os and P. Domen managed the analyses.

S. Michielse and P. Domen undertook the statistical analysis.

P. Domen managed the literature searches and wrote the first draft of the manuscript. All authors contributed to and have approved the final manuscript.

\section{Conflict of interest}

J. van Os has been an unrestricted research grant holder with, or has received financial compensation as an independent symposium speaker from, Eli Lilly, BMS Lundbeck, Organon, Janssen-Cilag, GlaxoSmithKline, AstraZeneca, Pfizer, and Servier.

M. Marcelis has received financial compensation as an independent symposium speaker from Eli Lilly and Janssen-Cilag.

All other authors report no biomedical financial interests or potential conflicts of interest.

\section{Acknowledgments}

We thank Truda Driesen and Inge Crolla for their coordinating roles in the data collection, as well as the G.R.O.U.P. investigators: René S. Kahn, Don H. Linszen, Jim van Os, Durk Wiersma; Richard Bruggeman, Wiepke Cahn, Lieuwe de Haan, Lydia Krabbendam, Inez Myin-Germeys.

\section{Appendix A. Supplementary data}

Supplementary data to this article can be found online at http:// dx.doi.org/10.1016/j.schres.2013.03.002.

\section{References}

Andersen, S.L., Tomada, A., Vincow, E.S., Valente, E., Polcari, A., Teicher, M.H., 2008 Preliminary evidence for sensitive periods in the effect of childhood sexual abuse on regional brain development. J. Neuropsychiatry Clin. Neurosci. 20 (3), 292-301.

Andreasen, N.C., Flaum, M., Arndt, S., 1992. The Comprehensive Assessment of Symptoms and History (CASH). An instrument for assessing diagnosis and psychopathology. Arch. Gen. Psychiatry 49 (8), 615-623.

Andreasen, N.C., Paradiso, S., O'Leary, D.S., 1998. "Cognitive dysmetria” as an integrative theory of schizophrenia; a dysfunction in cortical-subcortical-cerebellar circuitry. Schizophr. Bull. 24 (2), 203-218.

Andreasen, N.C., Pressler, M., Nopoulos, P., Miller, D., Ho, B.C., 2010. Antipsychotic dose equivalents and dose-years: a standardized method for comparing exposure to different drugs. Biol. Psychiatry 67 (3), 255-262.

Annett, M., 1970. A classification of hand preference by association analysis. Br. J. Psychol. 61 (3), 303-321.

APA, 2000. Diagnostic and Statistical Manual of Mental Disorders, 4th ed. American Psychiatric Association, Washington, DC.

Ardekani, B.A., Nierenberg, J., Hoptman, M.J., Javitt, D.C., Lim, K.O., 2003. MRI study of white matter diffusion anisotropy in schizophrenia. Neuroreport 14 (16), 2025-2029.

Bell, R.P., Foxe, J.J., Nierenberg, J., Hoptman, M.J., Garavan, H., 2011. Assessing white matter integrity as a function of abstinence duration in former cocaine-dependent individuals. Drug Alcohol Depend. 114 (2-3), 159-168.

Boos, H.B., Mandl, R.C., van Haren, N.E., Cahn, W., van Baal, G.C., Kahn, R.S., Hulshoff Pol, H.E., 2012. Tract-based diffusion tensor imaging in patients with schizophrenia and their non-psychotic siblings. Eur. Neuropsychopharmacol. http://dx.doi.org/ 10.1016/j.euroneuro.2012.05.015.

Bora, E., Yucel, M., Fornito, A., Pantelis, C., Harrison, B.J., Cocchi, L., Pell, G., Lubman, D.I., 2012. White matter microstructure in opiate addiction. Addict. Biol. 17 (1), 141-148.

Burns, J., Job, D., Bastin, M.E., Whalley, H., Macgillivray, T., Johnstone, E.C., Lawrie, S.M., 2003. Structural disconnectivity in schizophrenia: a diffusion tensor magnetic resonance imaging study. Br. J. Psychiatry 182, 439-443.

Cahn, W., Hulshoff Pol, H.E., Caspers, E., van Haren, N.E., Schnack, H.G., Kahn, R.S., 2004 Cannabis and brain morphology in recent-onset schizophrenia. Schizophr. Res. 67 (2-3), 305-307.

Camchong, J., Lim, K.O., Sponheim, S.R., Macdonald, A.W., 2009. Frontal white matter integrity as an endophenotype for schizophrenia: diffusion tensor imaging in monozygotic twins and patients' nonpsychotic relatives. Front. Hum. Neurosci. 3, 35.

Chaim, T.M., Schaufelberger, M.S., Ferreira, L.K., Duran, F.L., Ayres, A.M., Scazufca, M., Menezes, P.R., Amaro Jr., E., Leite, C.C., Murray, R.M., McGuire, P.K., Rushe, T.M. Busatto, G.F., 2010. Volume reduction of the corpus callosum and its relationship with deficits in interhemispheric transfer of information in recent-onset psychosis Psychiatry Res. 184 (1), 1-9.

Cheung, V., Cheung, C., McAlonan, G.M., Deng, Y., Wong, J.G., Yip, L., Tai, K.S., Khong P.L., Sham, P., Chua, S.E., 2008. A diffusion tensor imaging study of structural dysconnectivity in never-medicated, first-episode schizophrenia. Psychol. Med. 38 (6), 877-885.

Clark, K.A., Nuechterlein, K.H., Asarnow, R.F., Hamilton, L.S., Phillips, O.R., Hageman, N.S. Woods, R.P., Alger, J.R., Toga, A.W., Narr, K.L., 2011. Mean diffusivity and fractional anisotropy as indicators of disease and genetic liability to schizophrenia. J. Psychiatr. Res. 45 (7), 980-988.

DeLisi, L.E., 2008. The effect of cannabis on the brain: can it cause brain anomalies that lead to increased risk for schizophrenia? Curr. Opin. Psychiatry 21 (2), 140-150.

Ellison-Wright, I., Bullmore, E., 2009. Meta-analysis of diffusion tensor imaging studies in schizophrenia. Schizophr. Res. 108 (1-3), 3-10.

Friston, K.J., 1998. The disconnection hypothesis. Schizophr. Res. 30, 115-125.

Friston, K.J., Frith, C.D., 1995. Schizophrenia: a disconnection syndrome? Clin. Neurosci. 3 (2), 89-97.

Gasparotti, R., Valsecchi, P., Carletti, F., Galluzzo, A., Liserre, R., Cesana, B., Sacchetti, E., 2009. Reduced fractional anisotropy of corpus callosum in first-contact, antipsychotic drug-naive patients with schizophrenia. Schizophr. Res. 108 (1-3), 41-48. 
Goghari, V.M., Rehm, K., Carter, C.S., MacDonald III, A.W., 2007. Regionally specific cortical thinning and gray matter abnormalities in the healthy relatives of schizophrenia patients. Cereb. Cortex 17 (2), 415-424.

Habets, P., Marcelis, M., Gronenschild, E., Drukker, M., van Os, J., 2010. Reduced cortica thickness as an outcome of differential sensitivity to environmental risks in schizophrenia. Biol. Psychiatry 69 (5), 487-494.

Hao, Y., Yan, Q., Liu, H., Xu, L., Xue, Z., Song, X., Kaneko, Y., Jiang, T., Liu, Z., Shan, B., 2009. Schizophrenia patients and their healthy siblings share disruption of white matter integrity in the left prefrontal cortex and the hippocampus but not the anterior cingulate cortex. Schizophr. Res. 114 (1-3), 128-135

Ho, B.C., Andreasen, N.C., Ziebell, S., Pierson, R., Magnotta, V., 2011. Long-term antipsychotic treatment and brain volumes: a longitudinal study of first-episode schizophrenia. Arch. Gen. Psychiatry 68 (2), 128-137.

Hua, K., Zhang, J., Wakana, S., Jiang, H., Li, X., Reich, D.S., Calabresi, P.A., Pekar, J.J., van Zijl, P.C., Mori, S., 2008. Tract probability maps in stereotaxic spaces: analyses of white matter anatomy and tract-specific quantification. NeuroImage 39 (1), 336-347.

Hubl, D., Koenig, T., Strik, W., Federspiel, A., Kreis, R., Boesch, C., Maier, S.E., Schroth, G., Lovblad, K., Dierks, T., 2004. Pathways that make voices: white matter changes in auditory hallucinations. Arch. Gen. Psychiatry 61 (7), 658-668.

Hulshoff Pol, H.E., Schnack, H.G., Mandl, R.C., Brans, R.G., van Haren, N.E., Baare, W.F., van Oel, C.J., Collins, D.L., Evans, A.C., Kahn, R.S., 2006. Gray and white matter density changes in monozygotic and same-sex dizygotic twins discordant for schizophrenia using voxel-based morphometry. Neurolmage 31 (2), 482-488.

Jones, D.K., Cercignani, M., 2010. Twenty-five pitfalls in the analysis of diffusion MRI data. NMR Biomed. 23 (7), 803-820.

Kanaan, R.A., Kim, J.S., Kaufmann, W.E., Pearlson, G.D., Barker, G.J., McGuire, P.K., 2005. Diffusion tensor imaging in schizophrenia. Biol. Psychiatry 58 (12), 921-929.

Kanaan, R.A., Allin, M., Picchioni, M., Barker, G.J., Daly, E., Shergill, S.S., Woolley, J., McGuire, P.K., 2012. Gender differences in white matter microstructure. PLoS One 7 (6), e38272.

Kay, S.R., Fiszbein, A., Opler, L.A., 1987. The positive and negative syndrome scale (PANSS) for schizophrenia. Schizophr. Bull. 13 (2), 261-276.

Knochel, C., Oertel-Knochel, V., Schonmeyer, R., Rotarska-Jagiela, A., van de Ven, V., Prvulovic, D., Haenschel, C., Uhlhaas, P., Pantel, J., Hampel, H., Linden, D.E., 2012. Interhemispheric hypoconnectivity in schizophrenia: fiber integrity and volume differences of the corpus callosum in patients and unaffected relatives. NeuroImage 59 (2), 926-934.

Konrad, A., Winterer, G., 2008. Disturbed structural connectivity in schizophrenia primary factor in pathology or epiphenomenon? Schizophr. Bull. 34 (1), 72-92.

Konrad, A., Vucurevic, G., Musso, F., Stoeter, P., Dahmen, N., Winterer, G., 2009. ErbB4 genotype predicts left frontotemporal structural connectivity in human brain. Neuropsychopharmacology 34 (3), 641-650.

Kubicki, M., McCarley, R., Westin, C.F., Park, H.J., Maier, S., Kikinis, R., Jolesz, F.A Shenton, M.E., 2007. A review of diffusion tensor imaging studies in schizophrenia. J. Psychiatr. Res. 41 (1-2), 15-30.

Kubicki, M., Styner, M., Bouix, S., Gerig, G., Markant, D., Smith, K., Kikinis, R., McCarley, R.W., Shenton, M.E., 2008. Reduced interhemispheric connectivity in schizophreniatractography based segmentation of the corpus callosum. Schizophr. Res. 106 (2-3), 125-131.

Kyriakopoulos, M., Bargiotas, T., Barker, G.J., Frangou, S., 2008. Diffusion tensor imaging in schizophrenia. Eur. Psychiatry 23 (4), 255-273.

Lane, S.D., Steinberg, J.L., Ma, L., Hasan, K.M., Kramer, L.A., Zuniga, E.A., Narayana, P.A. Moeller, F.G., 2010. Diffusion tensor imaging and decision making in cocaine dependence. PLoS One 5 (7), e11591.

Lederbogen, F., Kirsch, P., Haddad, L., Streit, F., Tost, H., Schuch, P., Wust, S., Pruessner, J.C., Rietschel, M., Deuschle, M., Meyer-Lindenberg, A., 2011. City living and urban upbringing affect neural social stress processing in humans. Nature 474 (7352) 498-501.

Liao, Y., Huang, X., Wu, Q., Yang, C., Kuang, W., Du, M., Lui, S., Yue, Q., Chan, R.C., Kemp, G.J., Gong, Q., 2012. Is depression a disconnection syndrome? Meta-analysis of diffusion tensor imaging studies in patients with MDD. J. Psychiatry Neurosci. 37 (4), 110180.

Makris, N., Seidman, L.J., Ahern, T., Kennedy, D.N., Caviness, V.S., Tsuang, M.T. Goldstein, J.M., 2010. White matter volume abnormalities and associations with symptomatology in schizophrenia. Psychiatry Res. 183 (1), 21-29.

Marenco, S., Radulescu, E., 2010. Imaging genetics of structural brain connectivity and neural integrity markers. Neurolmage 53 (3), 848-856.

Maxwell, M., 1992. Family Interview for Genetic Studies (FIGS): Manual for FIGS. Clinical Neurogenetics Branch, Intramural Research Program, National Institute of Mental Health, Bethesda, MD.

McDonald, C., Bullmore, E.T., Sham, P.C., Chitnis, X., Wickham, H., Bramon, E., Murray, R.M., 2004. Association of genetic risks for schizophrenia and bipolar disorder with specific and generic brain structural endophenotypes. Arch. Gen. Psychiatry 61 (10), 974-984.

McIntosh, A.M., Moorhead, T.W., Job, D., Lymer, G.K., Munoz Maniega, S., McKirdy, J. Sussmann, J.E., Baig, B.J., Bastin, M.E., Porteous, D., Evans, K.L., Johnstone, E.C. Lawrie, S.M., Hall, J., 2008. The effects of a neuregulin 1 variant on white matter density and integrity. Mol. Psychiatry 13 (11), 1054-1059.

Menzler, K., Belke, M., Wehrmann, E., Krakow, K., Lengler, U., Jansen, A., Hamer, H.M. Oertel, W.H., Rosenow, F., Knake, S., 2011. Men and women are different: diffusion tensor imaging reveals sexual dimorphism in the microstructure of the thalamus, corpus callosum and cingulum. NeuroImage 54 (4), 2557-2562.

Mitelman, S.A., Torosjan, Y., Newmark, R.E., Schneiderman, J.S., Chu, K.W., Brickman, A.M., Haznedar, M.M., Hazlett, E.A., Tang, C.Y., Shihabuddin, L., Buchsbaum, M.S 2007. Internal capsule, corpus callosum and long associative fibers in good and poor outcome schizophrenia: a diffusion tensor imaging survey. Schizophr. Res. $92(1-3), 211-224$
Miyata, J., Yamada, M., Namiki, C., Hirao, K., Saze, T., Fujiwara, H., Shimizu, M., Kawada, R., Fukuyama, H., Sawamoto, N., Hayashi, T., Murai, T., 2010. Reduced white matter integrity as a neural correlate of social cognition deficits in schizophrenia. Schizophr. Res. 119 (1-3), 232-239.

Mori, S., Oishi, K., Jiang, H., Jiang, L., Li, X., Akhter, K., Hua, K., Faria, A.V., Mahmood, A. Woods, R., Toga, A.W., Pike, G.B., Neto, P.R., Evans, A., Zhang, J., Huang, H., Miller, M.I., van Zijl, P., Mazziotta, J., 2008. Stereotaxic white matter atlas based on diffusion tensor imaging in an ICBM template. Neurolmage 40 (2), 570-582.

Munoz Maniega, S., Lymer, G.K., Bastin, M.E., Marjoram, D., Job, D.E., Moorhead, T.W., Owens, D.G., Johnstone, E.C., McIntosh, A.M., Lawrie, S.M., 2008. A diffusion tensor MRI study of white matter integrity in subjects at high genetic risk of schizophrenia. Schizophr. Res. 106 (2-3), 132-139.

Narr, K.L., Hageman, N., Woods, R.P., Hamilton, L.S., Clark, K., Phillips, O., Shattuck, D.W., Asarnow, R.F., Toga, A.W., Nuechterlein, K.H., 2009. Mean diffusivity: a biomarker for CSF-related disease and genetic liability effects in schizophrenia. Psychiatry Res 171 (1), 20-32.

Navari, S., Dazzan, P., 2009. Do antipsychotic drugs affect brain structure? A systematic and critical review of MRI findings. Psychol. Med. 39 (11), 1763-1777.

Olabi, B., Ellison-Wright, I., McIntosh, A.M., Wood, S.J., Bullmore, E., Lawrie, S.M., 2011. Are there progressive brain changes in schizophrenia? A meta-analysis of structural magnetic resonance imaging studies. Biol. Psychiatry 70 (1), 88-96.

Patel, S., Mahon, K., Wellington, R., Zhang, J., Chaplin, W., Szeszko, P.R., 2011. A metaanalysis of diffusion tensor imaging studies of the corpus callosum in schizophrenia. Schizophr. Res. 129 (2-3), 149-155.

Peters, B.D., de Haan, L., Dekker, N., Blaas, J., Becker, H.E., Dingemans, P.M., Akkerman, E.M., Majoie, C.B., van Amelsvoort, T., den Heeten, G.J., Linszen, D.H., 2008. White matter fibertracking in first-episode schizophrenia, schizoaffective patients and subjects at ultra-high risk of psychosis. Neuropsychobiology 58 (1), 19-28.

Peters, B.D., Blaas, J., de Haan, L., 2010. Diffusion tensor imaging in the early phase of schizophrenia: what have we learned? J. Psychiatr. Res. 44 (15), 993-1004.

Price, G., Bagary, M.S., Cercignani, M., Altmann, D.R., Ron, M.A., 2005. The corpus callosum in first episode schizophrenia: a diffusion tensor imaging study. J. Neurol. Neurosurg. Psychiatry 76 (4), 585-587.

Price, G., Cercignani, M., Parker, G.J., Altmann, D.R., Barnes, T.R., Barker, G.J., Joyce, E.M., Ron, M.A., 2007. Abnormal brain connectivity in first-episode psychosis: a diffusion MRI tractography study of the corpus callosum. NeuroImage 35 (2), 458-466.

Rametti, G., Junque, C., Falcon, C., Bargallo, N., Catalan, R., Penades, R., Garzon, B., Bernardo, M., 2009. A voxel-based diffusion tensor imaging study of temporal white matter in patients with schizophrenia. Psychiatry Res. 171 (3), 166-176.

Rotarska-Jagiela, A., Schonmeyer, R., Oertel, V., Haenschel, C., Vogeley, K., Linden, D.E. 2008. The corpus callosum in schizophrenia-volume and connectivity changes affect specific regions. NeuroImage 39 (4), 1522-1532.

Seok, J.H., Park, H.J., Chun, J.W., Lee, S.K., Cho, H.S., Kwon, J.S., Kim, J.., 2007. White matter abnormalities associated with auditory hallucinations in schizophrenia: a combined study of voxel-based analyses of diffusion tensor imaging and structural magnetic resonance imaging. Psychiatry Res. 156 (2), 93-104.

Skelly, L.R., Calhoun, V., Meda, S.A., Kim, J., Mathalon, D.H., Pearlson, G.D., 2008. Diffusion tensor imaging in schizophrenia: relationship to symptoms. Schizophr. Res. 98 (1-3), 157-162.

Smieskova, R., Fusar-Poli, P., Allen, P., Bendfeldt, K., Stieglitz, R.D., Drewe, J., Radue, E.W., McGuire, P.K., Riecher-Rossler, A., Borgwardt, S.J., 2009. The effects of antipsychotics on the brain: what have we learnt from structural imaging of schizophrenia?-a systematic review. Curr. Pharm. Des. 15 (22), 2535-2549.

Smith, S.M., 2002. Fast robust automated brain extraction. Hum. Brain Mapp. 17 (3), $143-155$

Smith, S.M., Nichols, T.E., 2009. Threshold-free cluster enhancement: addressing problems of smoothing, threshold dependence and localisation in cluster inference. NeuroImage 44 (1), 83-98.

Smith, S.M., Jenkinson, M., Woolrich, M.W., Beckmann, C.F., Behrens, T.E., JohansenBerg, H., Bannister, P.R., De Luca, M., Drobnjak, I., Flitney, D.E., Niazy, R.K., Saunders, J., Vickers, J., Zhang, Y., De Stefano, N., Brady, J.M., Matthews, P.M. 2004. Advances in functional and structural MR image analysis and implementation as FSL. NeuroImage 23 (Suppl. 1), S208-S219.

Smith, S.M., Jenkinson, M., Johansen-Berg, H., Rueckert, D., Nichols, T.E., Mackay, C.E. Watkins, K.E., Ciccarelli, O., Cader, M.Z., Matthews, P.M., Behrens, T.E., 2006. Tract-based spatial statistics: voxelwise analysis of multi-subject diffusion data. Neurolmage 31 (4), 1487-1505.

Solowij, N., Yucel, M., Respondek, C., Whittle, S., Lindsay, E., Pantelis, C., Lubman, D.I., 2011. Cerebellar white-matter changes in cannabis users with and without schizophrenia. Psychol. Med. 41 (11), 2349-2359.

StataCorp, 2009. Stata Statistical Software: Release 12. StataCorp LP, College Station, TX

Steel, R.M., Bastin, M.E., McConnell, S., Marshall, I., Cunningham-Owens, D.G., Lawrie, S.M., Johnstone, E.C., Best, J.J., 2001. Diffusion tensor imaging (DTI) and proton magnetic resonance spectroscopy (1H MRS) in schizophrenic subjects and normal controls. Psychiatry Res. 106 (3), 161-170.

Sullivan, E.V., Adalsteinsson, E., Hedehus, M., Ju, C., Moseley, M., Lim, K.O., Pfefferbaum, A., 2001. Equivalent disruption of regional white matter microstructure in ageing healthy men and women. Neuroreport 12 (1), 99-104.

Szeszko, P.R., Robinson, D.G., Ashtari, M., Vogel, J., Betensky, J., Sevy, S., Ardekani, B.A., Lencz, T., Malhotra, A.K., McCormack, J., Miller, R., Lim, K.O., Gunduz-Bruce, H., Kane, J.M., Bilder, R.M., 2008. Clinical and neuropsychological correlates of white matter abnormalities in recent onset schizophrenia. Neuropsychopharmacology 33 (5), 976-984.

van der Gaag, M., Hoffman, T., Remijsen, M., Hijman, R., de Haan, L., van Meijel, B., van Harten, P.N., Valmaggia, L., de Hert, M., Cuijpers, A., Wiersma, D., 2006. The five-factor 
model of the positive and negative syndrome scale II: a ten-fold cross-validation of a revised model. Schizophr. Res. 85 (1-3), 280-287.

van Os, J., Linscott, R.J., 2012. Introduction: the extended psychosis phenotyperelationship with schizophrenia and with ultrahigh risk status for psychosis. Schizophr. Bull. 38 (2), 227-230.

van Os, J., Kenis, G., Rutten, B.P., 2010. The environment and schizophrenia. Nature 468 (7321), 203-212.

Walterfang, M., Wood, S.J., Velakoulis, D., Pantelis, C., 2006. Neuropathological, neurogenetic and neuroimaging evidence for white matter pathology in schizophrenia. Neurosci. Biobehav. Rev. 30 (7), 918-948.

Wang, F., Jiang, T., Sun, Z., Teng, S.L., Luo, X., Zhu, Z., Zang, Y., Zhang, H., Yue, W., Qu, M., Lu, T., Hong, N., Huang, H., Blumberg, H.P., Zhang, D., 2009. Neuregulin 1 genetic variation and anterior cingulum integrity in patients with schizophrenia and healthy controls. J. Psychiatry Neurosci. 34 (3), 181-186.

WHO, 1990. Composite International Diagnostic Interview (CIDI). World Health Organization, Geneva.
Woodruff, P.W., Phillips, M.L., Rushe, T., Wright, I.C., Murray, R.M., David, A.S., 1997. Corpus callosum size and inter-hemispheric function in schizophrenia. Schizophr. Res. 23 (3), 189-196.

Zalesky, A., Fornito, A., Seal, M.L., Cocchi, L., Westin, C.F., Bullmore, E.T., Egan, G.F. Pantelis, C., 2011. Disrupted axonal fiber connectivity in schizophrenia. Biol. Psychiatry 69 (1), 80-89.

Zou, L.Q., Xie, J.X., Yuan, H.S., Pei, X.L., Dong, W.T., Liu, P.C., 2008. Diffusion tensor imaging study of the anterior limb of internal capsules in neuroleptic-naive schizophrenia Acad. Radiol. 15 (3), 285-289.

Zuliani, R., Moorhead, T.W., Bastin, M.E., Johnstone, E.C., Lawrie, S.M., Brambilla, P., O'Donovan, M.C., Owen, M.J., Hall, J., McIntosh, A.M., 2011. Genetic variants in the ErbB4 gene are associated with white matter integrity. Psychiatry Res. 191 (2), 133-137. 